\title{
Estimation for Stochastic Nonlinear Systems with Randomly Distributed Time-Varying Delays and Missing Measurements
}

\author{
Yan Che, ${ }^{1,2}$ Huisheng Shu, ${ }^{1}$ and Xiu Kan ${ }^{1}$ \\ ${ }^{1}$ School of Information Science and Technology, Donghua University, Shanghai 201620, China \\ ${ }^{2}$ Department of Electronics and Information Engineering, Putian University, Putian 351100, China
}

Correspondence should be addressed to Huisheng Shu, hsshu@dhu.edu.cn

Received 21 June 2012; Accepted 6 September 2012

Academic Editor: Zidong Wang

Copyright $\odot 2012$ Yan Che et al. This is an open access article distributed under the Creative Commons Attribution License, which permits unrestricted use, distribution, and reproduction in any medium, provided the original work is properly cited.

\begin{abstract}
The estimation problem is investigated for a class of stochastic nonlinear systems with distributed time-varying delays and missing measurements. The considered distributed time-varying delays, stochastic nonlinearities, and missing measurements are modeled in random ways governed by Bernoulli stochastic variables. The discussed nonlinearities are expressed by the statistical means. By using the linear matrix inequality method, a sufficient condition is established to guarantee the mean-square stability of the estimation error, and then the estimator parameters are characterized by the solution to a set of LMIs. Finally, a simulation example is exploited to show the effectiveness of the proposed design procedures.
\end{abstract}

\section{Introduction}

In the past decades, estimation techniques have been extensively investigated in many complex dynamical processes of networks such as target tracking [1], advanced aircrafts, and manufacturing processes. A number of estimation methods have been proposed in the literature, most of them are under the assumption that the measurements always contain true signals with the disturbances and the noises, see for example, [2-9]. But, in practical applications, the measurements may contain missing measurements due to many reasons such as the sensor temporal failures, network congestion, multipath fading, and high maneuverability of the tracked targets. Because of the clear engineering signification, the estimation problems with missing measurements have received attention, see for example [10-22]. 
Recently, with the rapid development of networks, novel methods and flexible models have been devoted, but the research of missing measurements is still a challenge, and the Bernoulli-based distributed model has still been a hot approach to modeling the missing observation cases. For example, in [10], the missing probability for each sensor is governed by an individual random variable satisfying a certain probabilistic distribution over the interval [0 1 1]. Packet dropouts and communication delays are considered simultaneously in [12]. The variance-constrained dissipative control problem for a class of stochastic nonlinear systems with multiple degraded measurements in [13], where the degraded probability for each sensor is governed by an individual random variable satisfying a certain probabilistic distribution over a given interval. The $H_{\infty}$ filtering problem has been addressed in [20] for a class of nonlinear systems with randomly occurring incomplete information, where the considered incomplete information includes both the sensor saturations and the missing measurements, a regional sensor model has been designed to account for both the randomly occurring sensor saturation and missing measurement in a unified representation, based on this sensor model, a newfangled $H_{\infty}$ filter with a certain ellipsoid constraint has been researched such that the filtering error dynamics is locally mean-square asymptotically stable and the $H_{\infty}$-norm requirement is satisfied.

On the other hand, time delays are frequently encountered in real-world application such as communications, engineering, and biological systems. The occurrence of time delays may induce instability, oscillation, and poor performance. Consequently, research on timedelay systems has been a topic of recurring interest over the past decades. Current efforts can be classified into several categories, for example, simple delay and multiple delays [12], delay-independence $[23,24]$ and delay-dependence $[5,8,25-30]$, time-varying delays [31,32] and constant delays, retarded-type delay and neutral-type delay [30,33], and mixed delays $[34,35]$. However, in some applications, such as these systems connected over a wireless networks/or neural networks, as pointed out in [36], networks usually have a spatial extent due of the presence of a multitude of parallel pathways with a variety of axon sizes and lengths, and therefore the propagation delays can be distributed over a period of time, so it is essential to describe the distributed time delay under the probability framework as possible as. In this paper, the probability distribution of the time-vary delays are described for Itô type discrete-time stochastic distribution by a binary switching sequence satisfying the Bernoullidistributed model.

Motivated by the aforementioned discussions, in this paper, we model the stochastic nonlinearities, the missing measurements, and the distributed time-vary delays by Bernoulli distributed white sequence with known conditional probability distribution. We aim at designing a estimator such that, for all possible measurements missing and distributed timevary delays to obtain the estimation error system mean-square stable. The solvability of the addressed estimation problem can be expressed as the feasibility of a set of LMIs. Finally, a numerical simulation example is exploited to show the effectiveness of the results derived. The main contributions of this paper are summarized as the following: (1) a new estimation problem is studied for the stochastic nonlinear systems with both distributed time-vary delays and measurements missing phenomenon; (2) a mean-square stable performance is taken into consideration for the addressed stochastic nonlinear systems with distributed timevary delays and missing measurements.

The rest of this paper is organized as follows. Section 2 briefly introduces the problem under consideration. In Section 3, a sufficient condition is established such that, for the missing measurements, the randomly distributed time-varying delays and nonlinearities, 
the estimation error system is the mean-square stability. A numerical example is given in Section 4. This paper is concluded in Section 5.

Notations. The notation used here is fairly standard except where otherwise stated. $\mathbb{R}^{n}, \mathbb{R}^{n \times m}$, and $\mathbb{I}^{+}$denote, respectively, the $n$-dimension Euclidean space, the set of all $n \times m$ real matrices, and the set of nonnegative integers. $\left(\Omega, \mathcal{F},\left\{\mathcal{F}_{k}\right\}_{k \in \mathbb{I}^{+}}, \mathbb{P}\right)$ is complete filtered probability space, $\Omega$ is the sample space, $\mathcal{F}$ is the $\sigma$-algebra of subsets of the sample space, and $\mathbb{P}$ is the probability measure on $\mathbb{F} . \mathbb{E}\{x\}$ stands for the expectation of the stochastic variable $x$. $\operatorname{Prob}\{\cdot\}$ is used for the occurrence probability of the event ".". The superscript " $T$ " stands for matrix transposition. $P>0(P \geq 0)$ means that matrix $P$ is real symmetric and positive definite (positive semi-definite). $\lambda_{\min }(\cdot)$ denotes the minimum eigenvalue of a matrix. $I$ and 0 represent the identity matrix and the zero matrix with appropriate dimensions, respectively. $\operatorname{diag}\left\{X_{1}, X_{2}, \ldots, X_{n}\right\}$ stands for a block-diagonal matrix with matrices $X_{1}, X_{2}, \ldots, X_{n}$ on the diagonal. In symmetric block matrices or long matrix expressions, we use " $*$ " to represent a term, that is, induced by symmetry. Matrices, if their dimensions are not explicitly stated, are assumed to be compatible for algebraic operations.

\section{Problem Formulation and Preliminaries}

Consider the following class of stochastic nonlinear system with distributed time-varying delays:

$$
\begin{gathered}
x(k+1)=A x(k)+\kappa_{1}(k) B \sum_{m=-\tau(k)}^{-1} x(k+m)+\kappa_{2}(k) f(x(k))+E_{1} x(k) w(k), \\
y(k)=\kappa_{3}(k) C x(k)+E_{2} x(k) w(k), \\
z(k)=H_{1} x(k),
\end{gathered}
$$

where $x(k) \in \mathbb{R}^{n}$ is the state vector, $y(k) \in \mathbb{R}^{m}$ is the measured output vector, $z(k) \in \mathbb{R}^{q}$ is the signal to be estimated, $w(k)$ is a one-dimensional, zero-mean, Gaussian white noise sequence on a probability space $\left(\Omega, \mathcal{F},\left\{\mathcal{F}_{k}\right\}_{k \in \mathbb{I}^{+}}, \mathbb{P}\right)$ with $\mathbb{E}\left\{\omega^{2}(k)\right\}=1, A, B, C, E_{1}, E_{2}$, and $H_{1}$ are known real constant matrices with appropriate dimensions, $\tau(k)$ denoting time-varying delays are positive integers and bounded, namely, $0<\tau_{l} \leq \tau(k) \leq \tau_{u}$, the stochastic variables $\kappa_{1}(k) \in \mathbb{R}, \kappa_{2}(k) \in \mathbb{R}$, and $\kappa_{3}(k) \in \mathbb{R}$ are Bernoulli distributed white sequence taking the values of 0 and 1 with

$$
\begin{aligned}
& \operatorname{Prob}\left\{\kappa_{1}(k)=1\right\}=\mathbb{E}\left\{\kappa_{1}(k)\right\}:=\alpha_{1}, \\
& \operatorname{Prob}\left\{\kappa_{1}(k)=0\right\}:=1-\alpha_{1}, \\
& \operatorname{Prob}\left\{\kappa_{2}(k)=1\right\}=\mathbb{E}\left\{\kappa_{2}(k)\right\}:=\alpha_{2}, \\
& \operatorname{Prob}\left\{\kappa_{2}(k)=0\right\}:=1-\alpha_{2}, \\
& \operatorname{Prob}\left\{\kappa_{3}(k)=1\right\}=\mathbb{E}\left\{\kappa_{3}(k)\right\}:=\alpha_{3}, \\
& \operatorname{Prob}\left\{\kappa_{3}(k)=0\right\}:=1-\alpha_{3},
\end{aligned}
$$

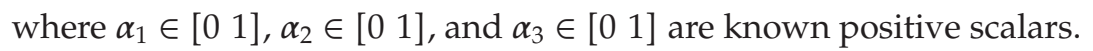


Remark 2.1. The nonlinear stochastic $f(x(k))$ is assumed to have the following for all $x(k)$ :

$$
\begin{gathered}
\mathbb{E}\{f(x(k)) \mid x(k)\}=0, \\
\mathbb{E}\left\{f(x(k)) f^{T}(x(k)) \mid x(k)\right\}=0, \quad k \neq j, \\
\mathbb{E}\left\{f(x(k)) f^{T}(x(k)) \mid x(k)\right\} \leq \sum_{i=1}^{q} \Pi_{i} x^{T}(k) \Phi_{i} x(k),
\end{gathered}
$$

where $q$ is a known nonnegative integer, $\Pi_{i}=\bar{\Pi}_{i} \bar{\Pi}_{i}^{T}, \Pi_{i}, \bar{\Pi}_{i}$, and $\Phi_{i}(i=1, \ldots, q)$ are known matrices with appropriate dimensions. For convenience, one assumes that $f(x(k))$ is unrelated with $\kappa_{1}(k), \kappa_{2}(k), \kappa_{3}(k)$, and $\omega(k)$.

In this paper, we aim at designing a linear estimator of the following structure:

$$
x_{f}(k+1)=A_{f} x_{f}(k)+A_{k} y(k), \quad \widehat{z}(k)=H_{2} x_{f}(k), \quad \widehat{z}(0)=0,
$$

where $x_{f} \in \mathbb{R}^{n}$ is the state estimate, $\widehat{z}(k)$ is the estimate output, $H_{2}$ is a known real constant matrix with appropriate dimension, and $A_{f}$ and $A_{k}$ are estimator parameters to be determined.

By defining $\widehat{x}(k)=\left[\begin{array}{ll}x^{T}(k) & x_{f}^{T}(k)\end{array}\right]^{T}$, we have the following augmented system:

$$
\begin{aligned}
\widehat{x}(k+1)= & \mathcal{A} \widehat{x}(k)+\bar{A} \widehat{x}(k)+B \sum_{m=-\tau(k)}^{-1} \widehat{x}(k+m)+\bar{B} \sum_{m=-\tau(k)}^{-1} \widehat{x}(k+m) \\
& +\mathcal{N} h(k)+\overline{\mathcal{N}} h(k)+\mathcal{\varepsilon} \widehat{x}(k) w(k),
\end{aligned}
$$

where

$$
\begin{gathered}
\mathcal{A}=\left[\begin{array}{cc}
A & 0 \\
\alpha_{3} A_{k} C & A_{f}
\end{array}\right], \quad \bar{A}=\left[\begin{array}{cc}
0 & 0 \\
\left(\kappa_{3}(k)-\alpha_{3}\right) A_{k} C & 0
\end{array}\right], \quad B=\left[\begin{array}{cc}
\alpha_{1} B & 0 \\
0 & 0
\end{array}\right], \\
\bar{B}=\left[\begin{array}{cc}
\left(\kappa_{1}(k)-\alpha_{1}\right) B & 0 \\
0 & 0
\end{array}\right], \quad \widehat{x}(k+i)=\left[\begin{array}{c}
x(k+i) \\
x_{f}(k+i)
\end{array}\right], \quad h(k)=\left[\begin{array}{c}
f(x(k)) \\
0
\end{array}\right], \\
\mathcal{E}=\left[\begin{array}{cc}
E_{1} & 0 \\
A_{k} E_{2} & 0
\end{array}\right], \quad \widehat{\mathcal{N}}=\left[\begin{array}{ll}
I & 0 \\
0 & 0
\end{array}\right], \quad \quad \mathcal{N}=\alpha_{2} \widehat{\mathcal{N}}, \quad \overline{\mathcal{N}}=\left(\kappa_{2}(k)-\alpha_{2}\right) \widehat{\mathcal{N}} .
\end{gathered}
$$

Observe the system $(2.10)$ and let $\widehat{x}(k ; \varphi)$ denote the state trajectory from the initial data $\widehat{x}(s)=\varphi(s)$ on $-\xi_{M} \leq s \leq-\xi_{m}$. Obviously, $\widehat{x}(k ; 0) \equiv 0$ is the trivial solution of system (2.10) corresponding to the initial data $\varphi=0$.

In what follows, we aim to design a linear estimator of the form (2.9) for system (2.1) such that, for all admissible randomly occurring distributed time-varying delays, missing measurements, stochastic nonlinearities, and estimation error system (2.10) is mean-square stable. 


\section{Main Results}

The following lemmas are essential in establishing our main results.

Lemma 3.1 (Schur Complement). There are constant matrices $\Upsilon_{1}, \Upsilon_{2}$, and $\Upsilon_{3}$ where $\Upsilon_{1}=\Upsilon_{1}^{T}$ and $\Upsilon_{2}=\Upsilon_{2}^{T}>0$, then $\Upsilon_{1}+\Upsilon_{3}^{T} \Upsilon_{2}^{-1} \Upsilon_{3}<0$ if and only if $\left[\begin{array}{cc}\Upsilon_{1} & \Upsilon_{3}^{T} \\ \Upsilon_{3} & -\Upsilon_{2}\end{array}\right]<0$.

Lemma 3.2. Let $\mathcal{W} \in \mathbb{R}^{n \times n}$ be a positive semidefinite matrix, $x_{i} \in \mathbb{R}^{n}$ be a vector, and $a_{i} \geq 0$ ( $i=$ $1,2, \ldots)$ be scalars. If the series concerned are convergent, then the following inequality holds [35]

$$
\left(\sum_{i=1}^{+\infty} a_{i} x_{i}\right)^{T} \mathcal{W}\left(\sum_{i=1}^{+\infty} a_{i} x_{i}\right) \leq\left(\sum_{i=1}^{+\infty} a_{i}\right) \sum_{i=1}^{+\infty} a_{i} x_{i}^{T} \mathcal{W} x_{i} .
$$

In the following theorem, Lyapunov stability theorem and a LMI-based method are combined together to deal with the stability analysis issue for the estimator design of the discrete-time stochastic nonlinear system with distributed time-varying delays and missing measurements. A sufficient condition is derived that ensures the solvability of the estimation problem.

Theorem 3.3. Given the estimator parameters $A_{f}$ and $A_{k}$ consider the estimation error system (2.10). If there exist positive definite matrices $P=P^{T}>0, Q=Q^{T}>0$, and positive scalars $\varpi_{i}>0(i=1,2, \ldots, q)$ such that the following matrix inequalities,

$$
\begin{aligned}
& {\left[\begin{array}{cccccccc}
-P & * & * & * & * & * & * & * \\
0 & -Q & * & * & * & * & * & * \\
P \mathcal{\varepsilon} & 0 & -P & * & * & * & * & * \\
\beta_{1} Q & 0 & 0 & -Q & * & * & * & * \\
P A & P B & 0 & 0 & -P & * & * & * \\
\beta_{3} P & 0 & 0 & 0 & 0 & -P & * & * \\
\beta_{4} \widehat{\Phi} & 0 & 0 & 0 & 0 & 0 & -\Xi & * \\
0 & \beta_{2} P \mathcal{F} & 0 & 0 & 0 & 0 & 0 & -P
\end{array}\right]<0,} \\
& \Delta=\left[\begin{array}{cc}
-\varpi_{i} I & * \\
P \widehat{\mathcal{M}}_{i} & -P
\end{array}\right]<0, \quad i=1,2, \ldots, q,
\end{aligned}
$$

hold, where

$$
\begin{gathered}
\beta_{1}=\left(\tau_{u}+\frac{1}{2}\left(\tau_{u}-\tau_{l}\right)\left(\tau_{u}+\tau_{l}-1\right)\right)^{1 / 2}, \quad \beta_{2}=\left(\alpha_{1}\left(1-\alpha_{1}\right)\right)^{1 / 2}, \\
\beta_{3}=\left(\alpha_{3}\left(1-\alpha_{3}\right)\right)^{1 / 2}, \quad \beta_{4}=\left(\alpha_{2}\right)^{1 / 2}, \quad \widehat{\Phi}=\left[\varpi_{1} \bar{\Phi}_{1}^{1 / 2}, \ldots, \varpi_{q} \bar{\Phi}_{q}^{1 / 2}\right]^{T}, \\
\bar{\Phi}_{i}=\left[\begin{array}{cc}
\Phi_{i} & 0 \\
0 & 0
\end{array}\right], \quad \bar{\Pi}_{i}=\left[\begin{array}{c}
\widehat{\pi}_{i} \\
\widehat{\pi}_{i}
\end{array}\right], \quad \Xi=\operatorname{diag}\left\{\varpi_{1} I, \ldots, \varpi_{q} I\right\}, \quad \mathcal{F}=\left[\begin{array}{ll}
B & 0 \\
0 & 0
\end{array}\right],
\end{gathered}
$$

then the estimation error system (2.10) is mean-square stable. 
Proof. Define the following Lyapunov functional candidate for system (2.10):

$$
\begin{aligned}
V(\widehat{x}(k), k)=\widehat{x}^{T}(k) P \widehat{x}(k)+\sum_{i=-\tau(k)}^{-1} \sum_{j=k+i}^{k-1} \widehat{x}^{T}(j) Q \widehat{x}(j) \\
+\sum_{i=-\tau_{u}} \sum_{j=i+1}^{-\tau_{l}-1} \sum_{n=k+j}^{k-1} \widehat{x}^{T}(n) Q \widehat{x}(n) .
\end{aligned}
$$
one has,

By calculating the difference of the Lyapunov functional (3.5), based on Lemma 3.2,

$$
\begin{aligned}
& \mathbb{E}\{\Delta V(\widehat{x}(k), k)\} \\
& =\mathbb{E}\{V(\widehat{x}(k+1), k+1) \mid \widehat{x}(k)\}-V\{(\widehat{x}(k), k)\} \\
& =\left[A \widehat{x}(k)+B \sum_{m=-\tau(k)}^{-1} \widehat{x}(k+m)\right]^{T} P\left[\mathcal{A} \widehat{x}(k)+B \sum_{m=-\tau(k)}^{-1} \widehat{x}(k+m)\right] \\
& +\mathbb{E}\left\{\left[\bar{B} \sum_{m=-\tau(k)}^{-1} \widehat{x}(k+m)\right]^{T} P\left[\bar{B} \sum_{m=-\tau(k)}^{-1} \hat{x}(k+m)\right]\right\}+\alpha_{3}(1-\alpha 3) \widehat{x}^{T}(k) P \widehat{x}(k) \\
& +\widehat{x}^{T}(k) \mathcal{E}^{T} P \varepsilon \widehat{x}(k)+\alpha_{2} \mathbb{E}\left\{h^{T}(k) \widehat{\mathcal{N}}^{T} P \widehat{\mathcal{N}} h(k)\right\}-\widehat{x}^{T}(k) P \widehat{x}(k) \\
& +\sum_{i=-\tau(k+1)}^{-1} \sum_{j=k+i+1}^{k} \widehat{x}^{T}(j) Q \widehat{x}(j)-\sum_{i=-\tau(k)}^{-1} \sum_{j=k+i}^{k-1} \widehat{x}^{T}(j) Q \widehat{x}(j) \\
& +\sum_{i=-\tau_{u}}^{-\tau_{l}-1} \sum_{j=i+1}^{-1}\left[\sum_{n=k+j+1}^{k}-\sum_{n=k+j}^{k-1}\right] \widehat{x}^{T}(n) Q \widehat{x}(n) \\
& =\left[A \widehat{x}(k)+B \sum_{m=-\tau(k)}^{-1} \widehat{x}(k+m)\right]^{T} P\left[A \widehat{x}(k)+B \sum_{m=-\tau(k)}^{-1} \widehat{x}(k+m)\right] \\
& +\mathbb{E}\left\{\left[\bar{B} \sum_{m=-\tau(k)}^{-1} \widehat{x}(k+m)\right]^{T} P\left[\bar{B} \sum_{m=-\tau(k)}^{-1} \widehat{x}(k+m)\right]\right\}+\alpha_{3}(1-\alpha 3) \widehat{x}^{T}(k) P \widehat{x}(k) \\
& +\widehat{x}^{T}(k) \mathcal{E}^{T} P \mathcal{E} \widehat{x}(k)+\alpha_{2} \mathbb{E}\left\{h^{T}(k) \widehat{\mathcal{N}}^{T} P \widehat{\mathcal{N}} h(k)\right\}-\widehat{x}^{T}(k) P \widehat{x}(k) \\
& +\sum_{i=-\tau(k+1)}^{-1}\left[\sum_{j=k+i+1}^{k-1} \widehat{x}^{T}(j) Q \widehat{x}(j)+\widehat{x}^{T}(k) Q \widehat{x}(k)\right] \\
& -\sum_{i=-\tau(k)}^{-1}\left[\sum_{j=k+i+1}^{k-1} \hat{x}^{T}(j) Q \widehat{x}(j)+\widehat{x}^{T}(k+i) Q \widehat{x}(k+i)\right] \\
& +\sum_{i=-\tau_{u}}^{-\tau_{l}-1} \sum_{j=i+1}^{-1}\left[\widehat{x}^{T}(k) Q \widehat{x}(k)-\widehat{x}^{T}(k+j) Q \widehat{x}(k+j)\right]
\end{aligned}
$$


Mathematical Problems in Engineering

$$
\begin{aligned}
& \leq\left[A \widehat{x}(k)+B \sum_{m=-\tau(k)}^{-1} \widehat{x}(k+m)\right]^{T} P\left[\mathcal{A} \widehat{x}(k)+B \sum_{m=-\tau(k)}^{-1} \widehat{x}(k+m)\right] \\
& +\mathbb{E}\left\{\left[\bar{B} \sum_{m=-\tau(k)}^{-1} \widehat{x}(k+m)\right]^{T}\left[\bar{B} \sum_{m=-\tau(k)}^{-1} \widehat{x}(k+m)\right]\right\}+\alpha_{3}(1-\alpha 3) \hat{x}^{T}(k) P \widehat{x}(k) \\
& +\widehat{x}^{T}(k) \mathcal{E}^{T} P \mathcal{\varepsilon} \widehat{x}(k)+\alpha_{2} \mathbb{E}\left\{h^{T}(k) \widehat{\mathcal{N}}^{T} P \widehat{\mathcal{N}} h(k)\right\}-\widehat{x}^{T}(k) P \widehat{x}(k) \\
& +\sum_{i=-\tau_{u}}^{-\tau_{l}-1} \sum_{j=k+i+1}^{k-1} \widehat{x}^{T}(j) Q \widehat{x}(j)+\sum_{i=-\tau_{l}}^{-1} \sum_{j=k+i+1}^{k-1} \widehat{x}^{T}(j) Q \widehat{x}(j) \\
& +\tau_{u} \widehat{x}^{T}(k) Q \widehat{x}(k)-\sum_{i=-\tau_{l}}^{-1} \sum_{j=k+i+1}^{k-1} \widehat{x}^{T}(j) Q \widehat{x}(j)-\sum_{i=-\tau(k)}^{-1} \widehat{x}^{T}(k+i) Q \widehat{x}(k+j) \\
& +\frac{1}{2}\left(\tau_{u}-\tau_{l}\right)\left(\tau_{u}+\tau_{l}-1\right) \widehat{x}^{T}(k) Q \widehat{x}(k)-\sum_{i=-\tau_{u}}^{-\tau_{l}-1} \sum_{j=k+i+1}^{k-1} \widehat{x}^{T}(j) Q \widehat{x}(j) \\
& =\left[A \widehat{x}(k)+B \sum_{m=-\tau(k)}^{-1} \widehat{x}(k+m)\right]^{T} P\left[A \widehat{x}(k)+B \sum_{m=-\tau(k)}^{-1} \widehat{x}(k+m)\right] \\
& +\mathbb{E}\left\{\left[\bar{B} \sum_{m=-\tau(k)}^{-1} \widehat{x}(k+m)\right]^{T} P\left[\bar{B} \sum_{m=-\tau(k)}^{-1} \widehat{x}(k+m)\right]\right\}+\alpha_{3}(1-\alpha 3) \widehat{x}^{T}(k) P \widehat{x}(k) \\
& +\widehat{x}^{T}(k) \mathcal{E}^{T} P \mathcal{E} \widehat{x}(k)+\alpha_{2} \mathbb{E}\left\{h^{T}(k) \widehat{\mathcal{N}}^{T} P \widehat{\mathcal{N}} h(k)\right\}-\widehat{x}^{T}(k) P \widehat{x}(k) \\
& +\tau_{u} \widehat{x}^{T}(k) Q \widehat{x}(k)-\sum_{i=-\tau(k)}^{-1} \widehat{x}^{T}(k+i) Q \widehat{x}(k+i) \\
& +\frac{1}{2}\left(\tau_{u}-\tau_{l}\right)\left(\tau_{u}+\tau_{l}-1\right) \widehat{x}^{T}(k) Q \widehat{x}(k) \\
& \leq\left[\mathcal{A} \widehat{x}(k)+B \sum_{m=-\tau(k)}^{-1} \widehat{x}(k+m)\right]^{T} P\left[\mathcal{A} \widehat{x}(k)+B \sum_{m=-\tau(k)}^{-1} \hat{x}(k+m)\right] \\
& +\mathbb{E}\left\{\left[\bar{B} \sum_{m=-\tau(k)}^{-1} \widehat{x}(k+m)\right]^{T} P\left[\bar{B} \sum_{m=-\tau(k)}^{-1} \widehat{x}(k+m)\right]\right\}+\alpha_{3}(1-\alpha 3) \widehat{x}^{T}(k) P \widehat{x}(k) \\
& +\widehat{x}^{T}(k) \mathcal{E}^{T} P \mathcal{\varepsilon} \widehat{x}(k)+\alpha_{2} \mathbb{E}\left\{h^{T}(k) \widehat{\mathcal{N}}^{T} P \widehat{\mathcal{N}} h(k)\right\}-\widehat{x}^{T}(k) P \widehat{x}(k) \\
& +\tau_{u} \widehat{x}^{T}(k) Q \widehat{x}(k)+\frac{1}{2}\left(\tau_{u}-\tau_{l}\right)\left(\tau_{u}+\tau_{l}-1\right) \widehat{x}^{T}(k) Q \widehat{x}(k) \\
& -\sum_{i=-\tau(k)}^{-1} \widehat{x}^{T}(k+i) Q \widehat{x}(k+i)
\end{aligned}
$$




$$
\begin{aligned}
\leq & {\left[\mathcal{A} \widehat{x}(k)+B \sum_{m=-\tau(k)}^{-1} \widehat{x}(k+m)\right]^{T} P\left[\mathcal{A} \widehat{x}(k)+\mathcal{B} \sum_{m=-\tau(k)}^{-1} \widehat{x}(k+m)\right] } \\
& +\mathbb{E}\left\{\left[\bar{B} \sum_{m=-\tau(k)}^{-1} \widehat{x}(k+m)\right]^{T} P\left[\bar{B} \sum_{m=-\tau(k)}^{-1} \widehat{x}(k+m)\right]\right\}+\alpha_{3}(1-\alpha 3) \widehat{x}^{T}(k) P \widehat{x}(k) \\
& +\widehat{x}^{T}(k) \mathcal{E}^{T} P \mathcal{E} \widehat{x}(k)+\alpha_{2} \mathbb{E}\left\{h^{T}(k) \widehat{\mathcal{N}}^{T} P \widehat{\mathcal{N} h}(k)\right\}-\widehat{x}^{T}(k) P \widehat{x}(k) \\
& +\tau_{u} \widehat{x}^{T}(k) Q \widehat{x}(k)+\frac{1}{2}\left(\tau_{u}-\tau_{l}\right)\left(\tau_{u}+\tau_{l}-1\right) \widehat{x}^{T}(k) Q \widehat{x}(k) \\
& -\tau_{u}^{-1}\left(\sum_{i=-\tau(k)}^{-1} \widehat{x}^{T}(k+i)\right) Q\left(\sum_{i=-\tau(k)}^{-1} \widehat{x}(k+i)\right) .
\end{aligned}
$$

From (2.8), it can be seen that

$$
\mathbb{E}\left\{h^{T}(k) \widehat{\mathcal{N}}^{T} P \widehat{\mathcal{N}} h(k)\right\} \leq \sum_{i=1}^{q}\left[\widehat{x}^{T}(k) \bar{\Phi}_{i} \widehat{x}(k)\right] \operatorname{tr}\left(\widehat{\mathcal{N}} \Pi_{i} \widehat{\mathcal{N}}^{T} P\right),
$$

where $\Pi_{i}:=\bar{\Pi}_{i} \bar{\Pi}_{i}^{T}$ with $\bar{\Phi}_{i}$ and $\bar{\Pi}_{i}$ defined in (3.4).

Furthermore,

$$
\begin{array}{r}
\mathbb{E}\left\{\left[\mathcal{B} \sum_{m=-\tau(k)}^{-1} \hat{x}(k+m)\right]^{T} P\left[\mathcal{B} \sum_{m=-\tau(k)}^{-1} \hat{x}(k+m)\right]\right\} \\
\leq \beta_{2}^{2} \sum_{m=-\tau(k)}^{-1} \hat{x}^{T}(k+m) \mathscr{F}^{T} P \mathcal{F} \sum_{m=-\tau(k)}^{-1} \widehat{x}(k+m),
\end{array}
$$

where $\beta_{2}$ is defined in (3.4).

From (3.6)-(3.8), one has

$$
\mathbb{E}\{\Delta V(\widehat{x}(k), k)\} \leq \mathbb{E}\left\{\eta^{T}(k) \Theta \eta(k)\right\},
$$

where $\eta(k)=\left[\widehat{x}^{T}(k), \sum_{i=-\tau(k)}^{-1} \hat{x}^{T}(k+i)\right]^{T}$ and

$$
\Theta=\left[\begin{array}{cc}
\Theta_{1} & A P ß \\
* & \Theta_{2}
\end{array}\right]
$$


where $\Theta_{1}=-P+\mathcal{E}^{T} P \mathcal{E}+\left(\tau_{u}+(1 / 2)\left(\tau_{u}-\tau_{l}\right)\left(\tau_{u}+\tau_{l}-1\right)\right) Q+\mathcal{A}^{T} P \mathscr{A}+\beta_{4}^{2} \sum_{i=1}^{q} \bar{\Phi}_{i} \operatorname{tr}\left(\widehat{\mathcal{N}} \Pi_{i} \widehat{\mathcal{N}}^{T} P\right)+$ $\beta_{3}^{2} P, \Theta_{2}=-\left(1 / \tau_{u}\right) Q+\mathbb{B}^{T} P \mathbb{B}+\beta_{2}^{2} \mathcal{F}^{T} P \mathcal{F}, \beta_{3}, \beta_{4}, \mathcal{F}$ are defined in (3.4).

From Lemma 3.1, (3.10) holds if and only if $\operatorname{tr}\left(\widehat{\mathcal{N}} \Pi_{i} \widehat{\mathcal{N}}^{T} P\right)$. Furthermore, by Lemma 3.1, one can obtain from (3.2), (3.3) that $\Theta<0$ and, subsequently,

$$
\mathbb{E}\{\Delta V(\widehat{x}(k), k)\}<-\lambda_{\min }(\Theta)|\widehat{x}(k)|^{2} .
$$

Thus, the augmented estimation system (2.10) is mean-square stable.

The following theorem is focused on the design of the desired estimation parameters $A_{f}$ and $A_{k}$ by using the results in Theorem 3.3.

Theorem 3.4. Consider the augmented estimation system (2.10) with given estimator parameters. If there exist positive-definite matrices $S=S^{T}>0, R=R^{T}>0, Q=Q^{T}>0$, matrices $\widetilde{A}_{f}, \widetilde{A}_{k}$, and positive scalars $\varpi_{i}>0,(i=1,2, \ldots, q)$ such that the following linear matrix inequalities holds

$$
\Gamma=\left[\begin{array}{ccccccccccccc}
-S & * & * & * & * & * & * & * & * & * & * & * & * \\
-S & -R & * & * & * & * & * & * & * & * & * & * & * \\
0 & 0 & -Q & * & * & * & * & * & * & * & * & * & * \\
S E_{1} & E_{1} & 0 & -S & * & * & * & * & * & * & * & * & * \\
\phi_{1} & \phi_{2} & 0 & -S & -R & * & * & * & * & * & * & * & * \\
\beta_{1} Q & \beta_{1} Q & 0 & 0 & 0 & -Q & * & * & * & * & * & * & * \\
S A & S A & \alpha_{1} S B & 0 & 0 & 0 & -S & * & * & * & * & * & * \\
\phi_{3} & \phi_{4} & \alpha_{1} R B & 0 & 0 & 0 & -S & -R & * & * & * & * & * \\
\beta_{3} S & \beta_{3} S & 0 & 0 & 0 & 0 & 0 & 0 & -S & * & * & * & * \\
\beta_{3} S & \beta_{3} R & 0 & 0 & 0 & 0 & 0 & 0 & -S & -R & * & * & * \\
\widetilde{\Phi} & \widetilde{\Phi} & 0 & 0 & 0 & 0 & 0 & 0 & 0 & 0 & -\Xi & * & * \\
0 & 0 & \beta_{2} S B & 0 & 0 & 0 & 0 & 0 & 0 & 0 & 0 & -R & * \\
0 & 0 & \beta_{2} R B & 0 & 0 & 0 & 0 & 0 & 0 & 0 & 0 & -S & -R
\end{array}\right]<0,
$$

hold, where $\alpha_{1}$ is defined in (2.2), $\beta_{1}, \beta_{2}, \beta_{3}$, and $\beta_{4}$ are defined in (3.4),

$$
\begin{gathered}
\tilde{\Phi}^{T}=\left[\beta_{4}\left[\varpi_{1} \Phi_{1}^{1 / 2}\right]^{T}, \ldots, \beta_{4}\left[\varpi_{q} \Phi_{q}^{1 / 2}\right]^{T}\right], \\
\phi_{1}=R E_{1}+\tilde{A}_{f} E_{2}, \quad \phi_{2}=R E_{1}+\tilde{A}_{f} E_{2}, \\
\phi_{3}=R A+\alpha_{2} \tilde{A}_{f} C+\tilde{A}_{k}, \quad \phi_{4}=R A+\alpha_{2} \tilde{A}_{f} C,
\end{gathered}
$$


then the estimator parameters are designed as

$$
A_{k}=X_{12}^{-1} \tilde{A}_{f}, \quad A_{f}=X_{12}^{-1} \tilde{A}_{k} S^{-1}\left(Y_{12}^{T}\right)^{-1},
$$

where $X_{12}, Y_{12}$ are any square and nonsingular matrices satisfying $X_{12} Y_{12}^{T}=I-R S^{-1}<0$, then the estimation error system (2.10) is mean-square stable.

Proof. Recall that our goal is to derive the expression of the estimator parameters from (2.9). To do this, we partition $P$ and $P^{-1}$ as

$$
P=\left[\begin{array}{cc}
R & X_{12} \\
X_{12}^{T} & X_{22}
\end{array}\right], \quad P^{-1}=\left[\begin{array}{ll}
S^{-1} & Y_{12} \\
Y_{12}^{T} & Y_{22}
\end{array}\right]
$$

where the partitioning of $P$ and $P^{-1}$ is compatible with that of $\mathcal{A}$ defined in (2.11), that is, $R \in R^{n \times n}, X_{12} \in R^{n \times n}, X_{22} \in R^{n \times n}, S \in R^{n \times n}, Y_{12} \in R^{n \times n}$, and $Y_{22} \in R^{n \times n}$. Define

$$
T_{1}=\left[\begin{array}{ll}
S^{-1} & I \\
Y_{12}^{T} & 0
\end{array}\right], \quad T_{2}=\left[\begin{array}{cc}
I & R \\
0 & X_{12}^{T}
\end{array}\right]
$$

which imply that $P T_{1}=T_{2}$ and $T_{1}^{T} P T_{1}=T_{1}^{T} T_{2}$.

By applying the congruence transformations $\operatorname{diag}\left\{T_{1}, I, T_{1}, I, T_{1}, T_{1}, I, \ldots, I, T_{1}\right\}$ and the congruence transformations $\operatorname{diag}\{S, I, I, S, I, I, S, I, S, I, I, \ldots, I, S, I\}$ to (3.2), we have (3.12).

Again, performing the congruence transformation $\operatorname{diag}\left\{I, T_{1}\right\}$ to (3.3) lead to (3.19)

$$
\left[\begin{array}{ccc}
-\varpi_{i} I & * & * \\
\widehat{\pi}_{i} & -S^{-1} & * \\
R \widehat{\pi}_{i}+X_{12} A_{k} \widehat{\pi}_{i} & -I & -R
\end{array}\right]<0, \quad i=1,2, \ldots, q .
$$

Then, one uses congruence transformation $\operatorname{diag}\{I, S, I\}$ to (3.19) and we have

$$
\left[\begin{array}{ccc}
-\varpi_{i} I & * & * \\
S \widehat{\pi}_{i} & -S & * \\
R \widehat{\pi}_{i}+X_{12} A_{k} \widehat{\pi}_{i} & -S & -R
\end{array}\right]<0, \quad i=1,2, \ldots, q .
$$

Furthermore, if (3.12) is feasible, we have $\left[\begin{array}{cc}-S & -S \\ -S & -R\end{array}\right]<0$ or $\left[\begin{array}{cc}-S^{-1} & I \\ I & R\end{array}\right]>0$.

It follows directly from $X X^{-1}=I$ that $I-R S^{-1}=X_{12} Y_{12}^{T}<0$. Hence, one can always find square and nonsingular $X_{12}$ and $Y_{12}$ [37]. Therefore, this completes the proof. 


\section{Numerical Example}

In this section, an example is presented to illustrate the usefulness and flexibility of the estimator design method developed in this paper. The system data of (2.1)-(2.9) are the following:

$$
\begin{array}{ccc}
A=\left[\begin{array}{cc}
0.15 & 0 \\
0.2 & 0.1
\end{array}\right], \quad B=\left[\begin{array}{cc}
0.09 & 0 \\
0 & 0.09
\end{array}\right], \quad C=\left[\begin{array}{cc}
1.2 & 0 \\
0 & 1.2
\end{array}\right], & \\
E_{1}=\left[\begin{array}{cc}
0.12 & 0 \\
0 & 0.12
\end{array}\right], \quad E_{2}=\left[\begin{array}{cc}
0.12 & 0 \\
0 & 0.12
\end{array}\right], & H_{1}=H_{2}=\left[\begin{array}{cc}
0.6 & 0 \\
0 & 0.6
\end{array}\right],
\end{array}
$$

where $n=q=2, \tau(k)=1+\left(1+(-1)^{k}\right), \tau_{l}=1, \tau_{u}=3$.

$f(x(k))$ describes the stochastic nonlinear function of the states in (2.1), which is bounded as follows:

$$
\mathbb{E}\left\{f(x(k)) f(x(k))^{T} \mid x(k)\right\}=\left[\begin{array}{l}
0.22 \\
0.22
\end{array}\right]\left[\begin{array}{c}
0.22 \\
0.22
\end{array}\right]^{T} x^{T}(k)\left[\begin{array}{cc}
0.11 & 0 \\
0 & 0.11
\end{array}\right] x(k) .
$$

Let $\alpha_{1}=0.2, \alpha_{2}=0.3$, and $\alpha_{3}=0.9$. Using Matlab LMI Toolbox to solve the LMIs in (3.12)(3.14), one has

$$
\begin{gathered}
S=\left[\begin{array}{cc}
0.6726 & -0.0035 \\
-0.0035 & 0.6563
\end{array}\right], \quad R=\left[\begin{array}{cc}
1.8796 & -0.0041 \\
-0.0041 & 1.8411
\end{array}\right], \\
Q=\left[\begin{array}{cc}
0.0668 & -0.0013 \\
-0.0013 & 0.0693
\end{array}\right], \quad \varpi_{1}=1.0776, \quad \varpi_{2}=1.3335
\end{gathered}
$$

Thus, we can calculate the estimator parameters as follows:

$$
A_{f}=\left[\begin{array}{cc}
0.1325 & 0.0486 \\
0.0462 & -0.1465
\end{array}\right], \quad A_{k}=\left[\begin{array}{cc}
-0.9144 & -0.1677 \\
-0.1684 & -0.8160
\end{array}\right]
$$

Remark 4.1. Seldom of the estimation literature explicitly introduce the effects of the estimators by the digits in the graphs, for example [18]. In this paper, some digits are marked in Figures 1-4. Figures 1-2 show the actual measurements and ideal measurements. Figures 3-4 plot the estimation errors. From these digits in the graphs, it can be seen that the designed estimator performs well.

\section{Conclusions}

In this paper, we research the estimation problem for a class of stochastic nonlinear systems with both the probabilistic distributed time-varying delays and missing measurements. The distributed time-varying delays and missing measurements are assumed to occur in random ways, and the occurring probabilities are governed by Bernoulli stochastic variables. A linear estimator is designed such that, for the admissible random distributed delays, the 


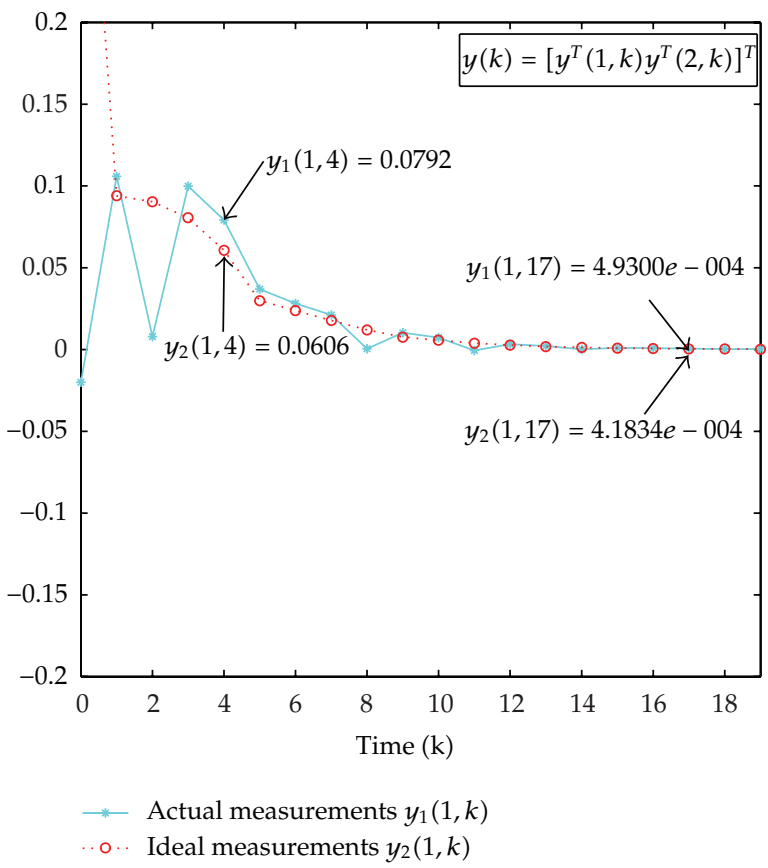

Figure 1: Actual Measurements $y_{1}(1, k)$ and ideal Measurements $y_{2}(1, k)$.

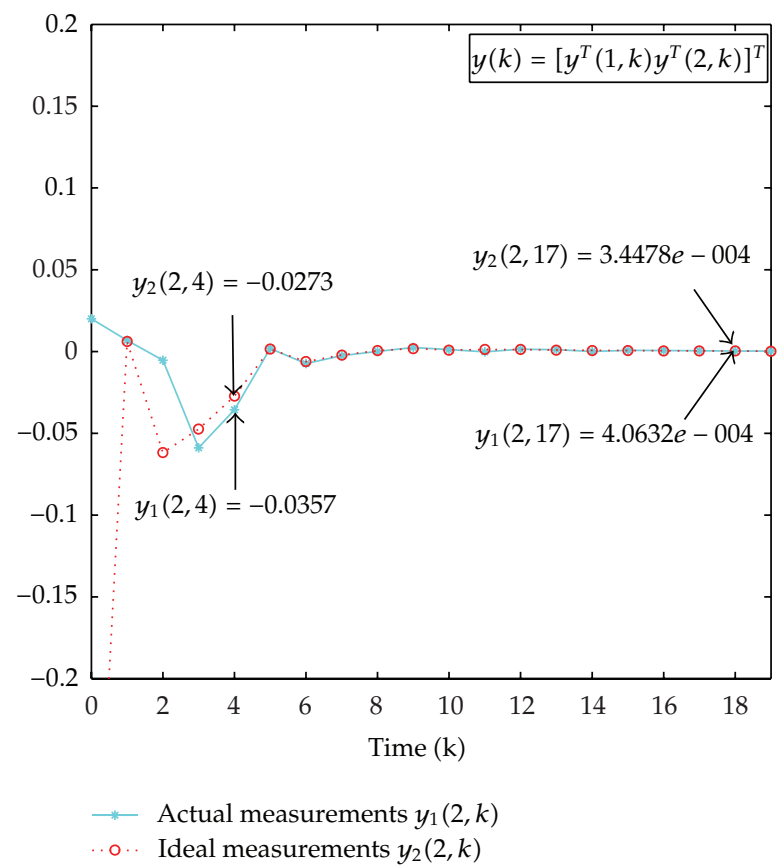

Figure 2: Actual Measurements $y_{1}(2, k)$ and ideal Measurements $y_{2}(2, k)$. 


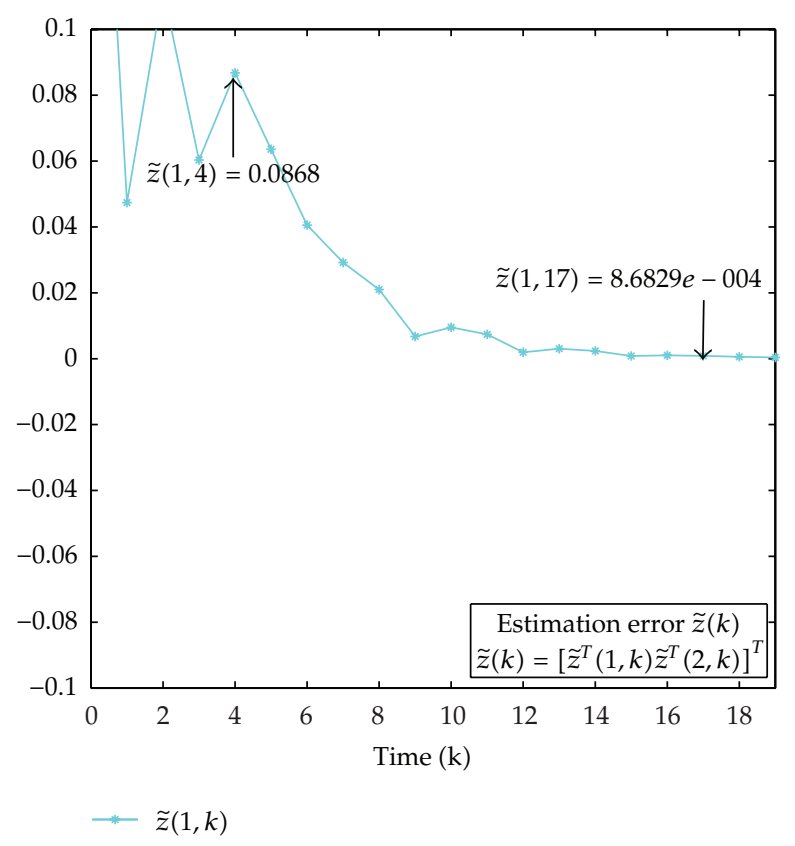

Figure 3: Estimation Errors $\widetilde{z}(1, k)$.

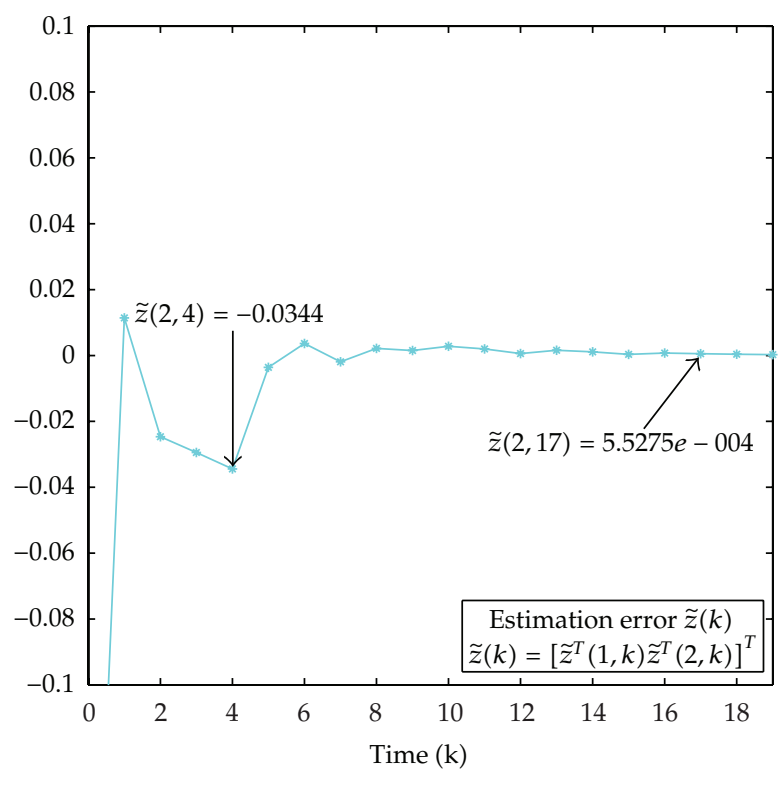

$\rightarrow-\tilde{z}(2, k)$

Figure 4: Estimation Errors $\widetilde{z}(2, k)$. 
stochastic disturbances, and the stochastic nonlinearities, the error dynamics of the estimation process is mean-square stable. At last, an illustrative example has been exploited to show the effectiveness of the proposed approach. In the future, we plan to consider the estimation problem with Markovian switching is in the finite-horizon case, and the nonlinearities are in more general forms.

\section{Acknowledgments}

This work was supported in part by the National Natural Science Foundation of China under Grant nos. 60974030 and 61104125, the Science and Technology Project of Education Department in Fujian Province under Grant no. JA11211, and the Science and Technology Innovation Platform "CAD/CAM Engineering Research Center of Universities of Fujian Province" which is set up in Putian University, Putian, Fujian Province, China.

\section{References}

[1] M. F. Bugallo and S. Xu, "Performance comparison of EKF and particle filtering methods for maneuvering targets," Digital Signal Processing, vol. 17, no. 4, pp. 774-786, 2007.

[2] C. Chen, H. Liu, and X. Guan, " $H_{\infty}$ filtering of time-delay T-S fuzzy systems based on piecewise Lyapunov-Krasovskii functional," Signal Processing, vol. 89, no. 10, pp. 1998-2005, 2009.

[3] O. Costa, "Stationary filter for linear minimum means quare error estimator of discretetime markovian jump systems," IEEE Transactions on Automatic Control, vol. 48, no. 8, pp. 1351-1356, 2002.

[4] H. Gao, J. Lam, L. Xie, and C. Wang, "New approach to mixed $H_{2} / H_{\infty}$ filtering for polytopic discretetime systems," IEEE Transactions on Signal Processing, vol. 53, no. 8, pp. 3183-3192, 2005.

[5] H. Gao and C. Wang, "A delay-dependent approach to robust $H_{\infty}$ filtering for uncertain discrete-time state-delayed systems," IEEE Transactions on Signal Processing, vol. 52, no. 6, pp. 1631-1640, 2004.

[6] H. Gao, J. Lam, C. Wang, and S. Xu, "Robust $H_{\infty}$ filtering for 2D stochastic systems," Circuits, Systems, and Signal Processing, vol. 23, no. 6, pp. 479-505, 2004.

[7] H. Gao, J. Lam, and C. Wang, "Robust energy-to-peak filter design for stochastic time-delay systems," Systems \& Control Letters, vol. 55, no. 2, pp. 101-111, 2006.

[8] W. Zhou, H. Su, and J. Chu, "Delay-dependent H1 filtering for singular Markovian jump time-delay systems," Signal Processing, vol. 90, no. 6, pp. 1815-1824, 2010.

[9] A. Subramanian and A. H. Sayed, "Multiobjective filter design for uncertain stochastic time-delay systems," IEE E Transactions on Automatic Control, vol. 49, no. 1, pp. 149-154, 2004.

[10] G. Wei, Z. Wang, and H. Shu, "Robust filtering with stochastic nonlinearities and multiple missing measurements," Automatica, vol. 45, no. 3, pp. 836-841, 2009.

[11] B. Shen, Z. Wang, H. Shu, and G. Wei, “On nonlinear $H_{\infty}$ filtering for discrete-time stochastic systems with missing measurements," IEEE Transactions on Automatic Control, vol. 53, no. 9, pp. 2170-2180, 2008.

[12] G. Wei, Z. Wang, X. He, and H. Shu, "Filtering for networked stochastic time-delay systems with sector nonlinearity," IEEE Transactions on Circuits and Systems-II, vol. 56, no. 1, pp. 71-75, 2009.

[13] Z. Wang, J. Lam, L. Ma, Y. Bo, and Z. Guo, "Variance-constrained dissipative observer-based control for a class of nonlinear stochastic systems with degraded measurements," Journal of Mathematical Analysis and Applications, vol. 377, no. 2, pp. 645-658, 2011.

[14] Z. Wang, F. Yang, D. W. C. Ho, and X. Liu, "Robust $H^{\infty}$ filtering for stochstic time-delay systems with missing measurements," IEEE Transactions on Signal Processing, vol. 54, no. 7, pp. 2579-2587, 2006.

[15] B. Shen, Z. Wang, and Y. S. Hung, "Distributed $H_{\infty}$-consensus filtering in sensor networks with multiple missing measurements: the finite-horizon case," Automatica, vol. 46, no. 10, pp. 1682-1688, 2010.

[16] Q. Ling and M. D. Lemmon, "Optimal dropout compensation in networked control systems," in Proceedings of the IEEE Conference on Decosion and Control, pp. 670-675, Honolulu, Hawaii, USA, 2003. 
[17] B. Sinopoli, L. Schenato, M. Franceschetti, K. Poolla, M. I. Jordan, and S. S. Sastry, "Kalman filtering with intermittent observations," IEEE Transactions on Automatic Control, vol. 49, no. 9, pp. 1453-1464, 2004.

[18] Y. Che, H. Shu, and Z. Wang, "Nonlinear systems filtering with missing measurements and randomly distributed delays," Journal of Harbin University of Science and Technology, vol. 16, no. 6, pp. 36-41, 2011.

[19] Z. Wang, B. Shen, H. Shu, and G. Wei, "Quantized H-infinity control for nonlinear stochastic timedelay systems with missing measurements," IEEE Transactions on Automatic Control, vol. 57, no. 6, pp. 1431-1444, 2012.

[20] Z. Wang, B. Shen, and X. Liu, " $H_{\infty}$ filtering with randomly occurring sensor saturations and missing measurements," Automatica, vol. 48, no. 3, pp. 556-562, 2012.

[21] B. Shen, Z. Wang, J. Liang, and Y. Liu, "Recent advances on filtering and control for nonlinear stochastic complex systems with incomplete information: a survey," Mathematical Problems in Engineering, vol. 2012, Article ID 530759, 16 pages, 2012.

[22] H. Dong, Z. Wang, and H. Gao, "Distributed filtering for a class of time-varying systems over sensor networks with quantization errors and successive packet dropouts," IEEE Transactions on Signal Processing, vol. 60, no. 6, pp. 3164-3173, 2012.

[23] V. Kapila and W. M. Haddad, "Memoryless H1 controllers for discrete-time systems with time delay," Automatica, vol. 34, no. 8, pp. 1141-1144, 1998.

[24] J. H. Kim and H. B. Park, " $H^{\infty}$ state feedback control for generalized continuous/discrete time-delay system," Automatica, vol. 35, no. 8, pp. 1443-1451, 1999.

[25] C. E. de Souza and X. Li, "Delay-dependent robust $H_{\infty}$ control of uncertain linear state-delayed systems," Automatica, vol. 35, no. 7, pp. 1313-1321, 1999.

[26] E. Fridman and U. Shaked, "A descriptor system approach to $H_{\infty}$ control of linear time-delay systems," IEEE Transactions on Automatic Control, vol. 47, no. 2, pp. 253-270, 2002.

[27] E. Fridman and U. Shaked, "An improved stabilization method for linear time-delay systems," IEEE Transactions on Automatic Control, vol. 47, no. 11, pp. 1931-1937, 2002.

[28] Y. S. Moon, P. Park, W. H. Kwon, and Y. S. Lee, “Delay-dependent robust stabilization of uncertain state-delayed systems," International Journal of Control, vol. 74, no. 14, pp. 1447-1455, 2001.

[29] Y. Zhang, S. Xu, Y. Zou, and J. Lu, "Delay-dependent robust stabilization for uncertain discrete-time fuzzy Markovian jump systems with mode-dependent time delays," Fuzzy Sets and Systems, vol. 164, no. 1, pp. 66-81, 2011.

[30] Z.-W. Liu and H.-G. Zhang, "Delay-dependent stability for systems with fast-varying neutral-type delays via a PTVD compensation," Acta Automatica Sinica, vol. 36, no. 1, pp. 147-152, 2010.

[31] D. Yue and H. Li, "Synchronization stability of continuous/discrete complex dynamical networks with interval time-varying delays," Neurocomputing, vol. 73, pp. 4809-6819, 2010.

[32] Y. Liu, Z. Wang, and X. Liu, "Robust $H_{\infty}$ filtering for discrete nonlinear stochastic systems with timevarying delay," Journal of Mathematical Analysis and Applications, vol. 341, no. 1, pp. 318-336, 2008.

[33] S. Xu, J. Lam, and C. Yang, " $H_{\infty}$ and positive-real control for linear neutral delay systems," IEEE Transactions on Automatic Control, vol. 46, no. 8, pp. 1321-1326, 2001.

[34] R. Rakkiyappan and P. Balasubramaniam, "Delay-probability-distribution-dependent stability of uncertain stochastic genetic regulatory networks with mixed time-varying delays: an LMI approach," Nonlinear Analysis. Hybrid Systems, vol. 4, no. 3, pp. 600-607, 2010.

[35] Y. Liu, Z. Wang, and X. Liu, "State estimation for discrete-time Markovian jumping neural networks with mixed mode-dependent delays," Physics Letters A, vol. 372, no. 48, pp. 7147-7155, 2008.

[36] H. Shu, Z. Wang, and Z. Lü, "Global asymptotic stability of uncertain stochastic bi-directional associative memory networks with discrete and distributed delays," Mathematics and Computers in Simulation, vol. 80, no. 3, pp. 490-505, 2009.

[37] C. Scherer, P. Gahinet, and M. Chilali, "Multiobjective output-feedback control via LMI optimization," IEEE Transactions on Automatic Control, vol. 42, no. 7, pp. 896-911, 1997. 


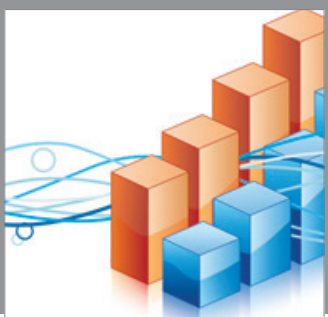

Advances in

Operations Research

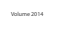

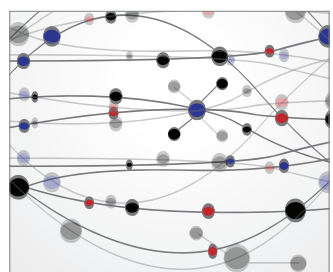

\section{The Scientific} World Journal
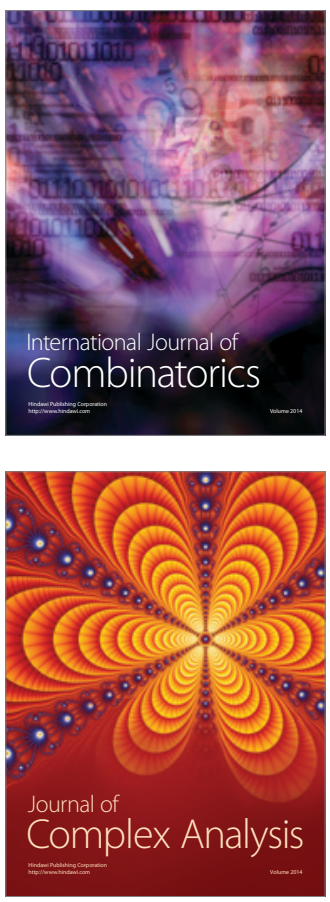

International Journal of

Mathematics and

Mathematical

Sciences
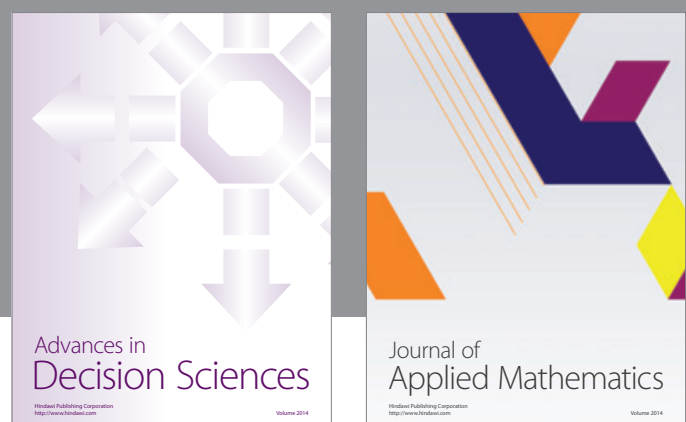

Journal of

Applied Mathematics
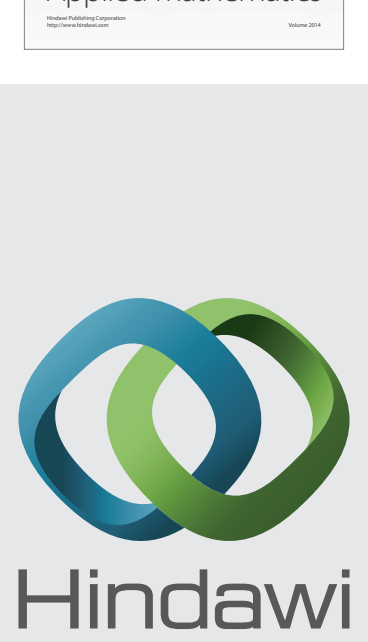

Submit your manuscripts at http://www.hindawi.com
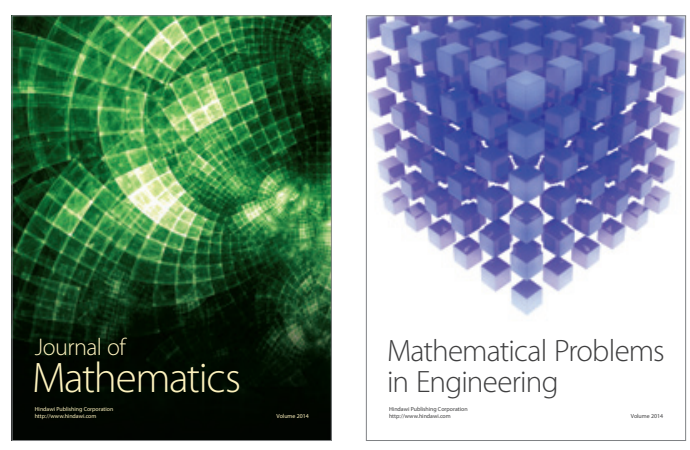

Mathematical Problems in Engineering
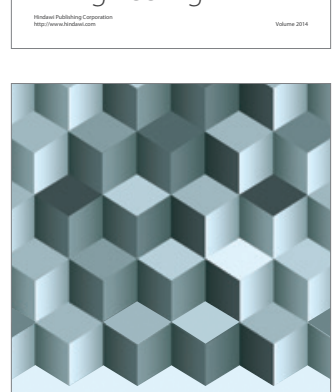

Journal of

Function Spaces
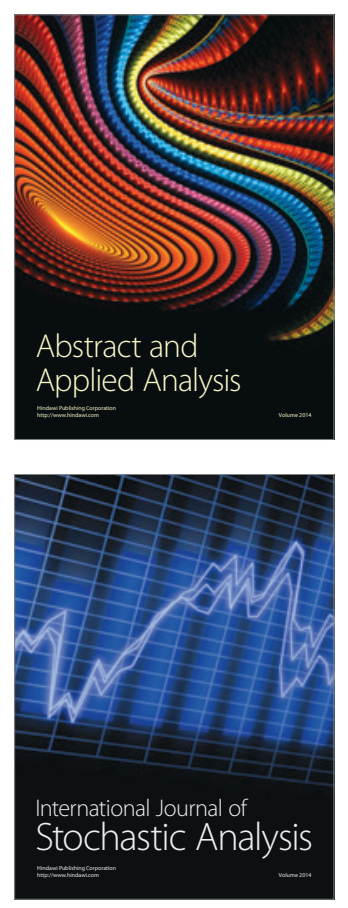

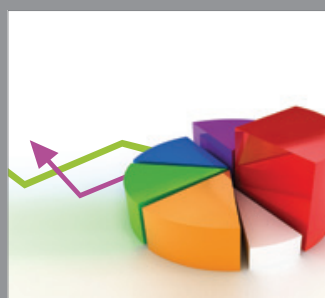

ournal of

Probability and Statistics

Promensencen
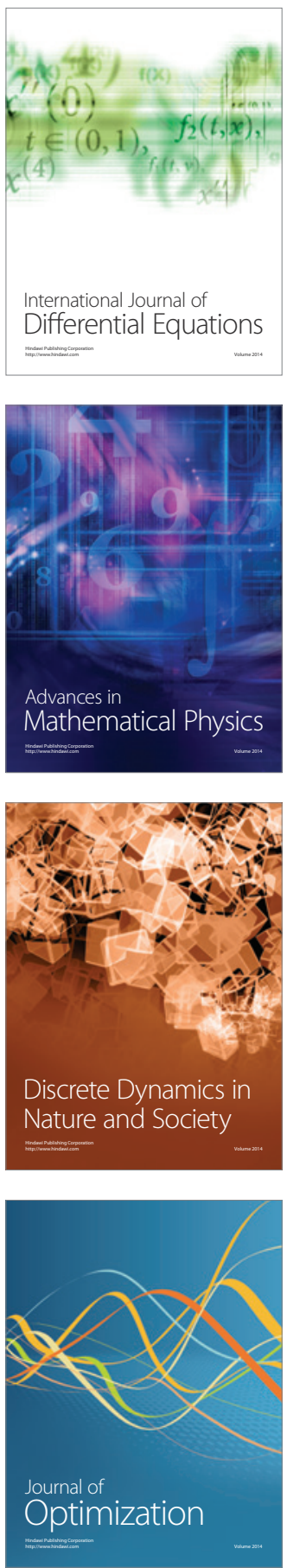